\title{
MEDICINA Y DERECHO \\ EL CONTROL DE LA VIDA Y LA MUERTE DEL INDIVIDUO, DESDE LA NORMA*
}

\section{Yolanda M. Guerra García Ph.D.**}

Fecha de recibido: 19 de marzo de 2012

Fecha de Aprobación: 21 de junio de 2012

Artículo resultado de investigación

¿A dónde iremos a parar? Si ya no solamente la vida, sino como vivirla y cómo morirla, son decisiones que le pertenecen exclusivamente al Estado.

Yolanda Guerra

\begin{abstract}
Resumen
Este artículo desarrolla la idea de cómo el Derecho hace parte de absolutamente todos los estadios en la vida de un individuo; todo, en relación con la vida, la muerte y lo que sucede en el intermedio está reglado por el Derecho. Todo está juridizado por el derecho, a través de normas: la vida y la muerte, la enfermedad, los tratamientos médicos y las posiciones éticas que debe asumir el médico frente a su paciente. Cuál es la conveniencia de que personas imperfectas que hacen las normas con intereses particulares y teniendo que pagar a quienes los subieron al poder diversos tipos de favores, sean quienes propongan los procedimientos y decisiones que deben tomarse en casos de vital importancia en la vida de las personas, como por ejemplo el caso de la eutanasia? Podría la ética o la bioética tener mayor relevancia en los casos importantes en donde la norma que regula la gerencia de la vida (biojurídica) se queda corta sobre todo en las discusiones biopolíticas?
\end{abstract}

\section{Palabras clave}

Eutanasia, Ética, biopolítica, biojurídica, bioética, Derecho.

\section{MEDICINE AND LAW \\ THE CONTROL OF LIFE AND DEATH OF AN INDIVIDUAL THROUGH THE LAW}

\begin{abstract}
This article develops the idea of how Law in general is everywhere one looks at. It makes a part of every single aspect of life, death, and everything in between: Everything is
\end{abstract}

\footnotetext{
Este artículo es resultado de una investigación sobre Bioética, trasplante de órganos y legislación comparada que a la fecha dirigida la Doctora Yolanda M. Guerra, en la Facultad de Educación y Humanidades de la Universidad Militar Nueva Granada.

** Yolanda M. Guerra docente investigador de la Facultad de Educación y Humanidades de la Universidad Militar Nueva Granada. Directora del grupo de investigación Liderazgo, Categoría A1 en Colciencias. Yolanda.guerra@unimilitar.edu.co
} 
somehow juridicized even before we are born: Illness, life, death, medical treatments and the ethical positions of the medical doctors before their patients. What is the convenience that imperfect people with particular interests make laws that regulate every single aspect of our lives and deaths, such as the case of euthanasia? Could somehow ethics or bioethics, be more appropriate to regulate the discussion in the cases of relevant importance of biojuridical and biopolitical scenarios?

\title{
Key words
}

Euthanasia, biopolítics, biojuridics, bioetics, Law.

\section{MEDICINA E DIREITO, O CONTROLE DA VIDA E MORTE DO INDIVÍDUO DESDE A NORMA}

\begin{abstract}
Resumo
Este artigo desenvolve a idéia de como a lei faz parte de absolutamente todas as fases na vida de um indivíduo, particularmente em relação à vida, morte eo que acontece no meio é regulamentada por lei. Tudo é judicializado por lei, através de regras: vida e morte, doenças, tratamentos médicos e posições éticas que devem assumir o médico na frente de seu paciente. Qual é o interesse de pessoas imperfeitas que fazem as regras com interesses particulares e ter que pagar para aqueles que chegaram ao poder vários tipos de favores, são defensores dos procedimentos e decisões a serem tomadas em casos de importância vital na vida do indivíduos, como para a eutanásia? Could ética ou bioética tem maior relevância em casos importantes em que a regra que regula a gestão da vida (biojurídica) está aquém, especialmente nas discussões bio-políticos?
\end{abstract}

\section{Palabra-Chave}

Eutanásia, biopolítica, biojuridics e bioetics, Direito.

\section{METODOLOGÍA E HIPÓTESIS}

La investigación que da origen a este documento, tiene un método de conocimiento interpretativo y analítico; basado en el desarrollo de un seminario profesoral que ya lleva tres años, en el cual se trabajan en cada sesión los autores más relevantes en torno a temas como la biopolítica y la biojurídica. Se ha trabajado a Michel Foucault, Giorgio Agamben, Hannah Arendt, Donna Haraway, Antonio Negri, Feliz Guattari y Jan Broekman, entre otros. Allí se ha puesto de manifiesto una verdad fundamental que hace parte de la hipótesis de este artículo: todas las actividades del hombre están regladas por la norma jurídica y por el derecho a través no solamente de la ley sino además de relaciones de poder donde el Estado prevalece e impone su voluntad por encima de la conveniencia del ser humano, teniendo además en cuenta un factor preponderante y de vital importancia: la economía y el presupuesto del Estado, sobre todo en cuestiones de salud, enfermedad, muerte y vida.

\section{INTRODUCCIÓN}

El Derecho hace parte de todo, en relación con la vida, la muerte y lo que sucede en el intermedio con las personas. La vida del individuo actual en pleno siglo XXI, en todos los países del mundo 
está reglada por el Derecho. Desde el aspecto más diminuto, como lo puede ser: cuándo es ilegal sacar un carro a la calle, hasta lo más trascendente como pudiera ser la idea de cuándo es considerada persona, un ser humano o cuando deja de serlo. Todo está regulado por el derecho, a través de normas. Ello y otros aspectos como la tecnología y el desarrollo, han creado un desplazamiento de valores fundamentales en la vida y en la sociedad. La relación médico paciente ha desplazado también su centro de gravedad, hacia la institución económica del Estado llamada medicina y el tratamiento de la enfermedad y la muerte como hechos carentes de emoción y de sentimiento.

No es algo bueno, al contrario. La deshumanización de la medicina, la economía de la enfermedad y el exceso de normas ha generado problemas de difícil solución. Además la norma ya no es respetada en Colombia. No por tener la Constitución política más extensa del mundo, se garantiza de ninguna manera el cumplimiento ni el respeto, siquiera por los derechos fundamentales del hombre (Tirado, 2011). La Constitución de Estados Unidos tiene 7 artículos, vigentes desde 1776, hace más de 250 años y el porcentaje de americanos que cumple la constitución, es mucho mayor a la de colombianos; cuya gran mayoría no saben qué hacer frente a la redacción de tanta norma confusa e inocua, para la muestra un ejemplo: artículo 22 de la CN "la paz es un derecho y un deber de obligatorio cumplimiento". ${ }^{1}$. El exceso de normas sobre la gerencia de la vida, se ha vuelto cuestión de semántica, como diría Roland Barthes. (1953).

\section{VIDA, MUERTE, ENFERMEDAD Y NORMA}

Los hombres hemos aprendido Más o menos a vivir culturalmente, Pero todavía la norma nos obliga a Morir como animales.

Gunther Anders

1 Constitución Política de Colombia, artículo 22
Regular aspectos tan relevantes como la vida y la muerte, la enfermedad, los tratamientos médicos y las posiciones éticas que debe asumir el médico frente a su paciente, es un desatino, que haría parte mejor no de la órbita del Derecho sino la órbita de la conciencia humana individual y por qué no decirlo, también espiritual. La vida del hombre no es solamente un zoe, como lo entendieron los Griegos, y que desarrollo siglos después Agamben (2009). Y quizá va más allá también del Bios.

En el interior del hombre hay una partícula divina trascendente de la cual ya hablaban los orientales hace siglos; incluso ya descubierta por los científicos no religiosos (Hamer, 2004) ubicada en el hueso sacro, (Sagrado para los Griegos) que hace que la vida no se limite al cuerpo físico intrascendente que se acaba con la muerte. Por ello legislar sobre la eutanasia o la enfermedad (y en general sobre cualquier cosa sin la debida responsabilidad y objetividad) en un país como Colombia es un verdadero peligro.

Pero el hombre y en concreto el Colombiano, ha querido crear normas sobre todo; hasta cómo educar a los individuos y de qué "llenarles" el cerebro. Por eso en 1968 en Colombia, se produce una reforma que antecedió la de 1992, dejando asignaturas vitales por fuera del pensum del colombiano, con el argumento de que "sobraban frente a los verdaderos retos de la vida. De allí para adelante todas las generaciones se educaron sin ética, sin valores, sin civismo, sin educación y buenas maneras, sin urbanidad, sin ciudadanía y patriotismo (Guerra, Y. 2009). Efectivamente esta enorme carencia, tiene sus consecuencias en los índices de criminalidad actuales, y se ve hoy en día reflejado en cada rostro que encontramos al voltear la esquina. En cada homicida de Transmilenio, en cada intolerante que maneja un carro en la calle, en cada uno de nosotros en general y en cualquier aspecto de la vida y de los roles que tenemos que asumir.

Todo tiene su norma en el Derecho actual desde que se nace hasta que se muere, pasando por la enfermedad y el cómo y dónde morir, hasta qué hacer con el remanente que un día tuvo 
vida, todo está reglado minuciosamente por el Estado. Sin importar si se trata de un Estado económicamente desarrollado o de los llamados del tercer mundo (Díaz, 1990). Colombia incluso ya hallenado los vacíos existentes sobre los protocolos de inhumación y exhumación, que antes no eran exegéticos en el derecho colombiano. Ahora hasta cargar un cadáver en un carro, sin los debidos permisos y actas, puede ser un delito en Colombia.

En cuanto a la salud, la situación es aún más desesperada. Comenzando por el Artículo 49 de la Constitución: "La atención de la salud y el saneamiento ambiental son servicios públicos a cargo del Estado. Se garantiza a todas las personas el acceso a los servicios de promoción, protección y recuperación de la salud". ¿Cómo se "garantiza" la atención de la salud en la vida real? Con la Ley 100 de 1993, conocida también como la Ley de la muerte en Colombia. Reiterando las apreciaciones de Roland Barthes, (1953) A veces las cosas más trascendentes y serias de la vida, se vuelven solo cuestión de semántica. (Barthes, 1953)

En lo atinente a la función de la medicina, el centro de gravedad se ha desplazado de lo que un día fue una relación médico - paciente a lo que hoy en día es una relación Estado-instituciones médicas. Lo más perverso de este "avance" de paradigma, es que el Derecho, es decir la norma, está hecha por personas imperfectas, seres falibles guiados por intereses particulares que deben atender primero las cuotas políticas y las deudas adquiridas con quienes los llevaron al poder. El interés general, casi nunca resulta verdaderamente reflejado en esas normas. Acá vale la pena recordar al canciller de hierro Otto Von Bismark, a quien se le atribuye la frase: Con las salchichas pasa como con las leyes, si uno observa cómo es que se hacen, les perdería todo el respeto y les cogería aversión. La cantidad de aberraciones en la creación de las unas y de las otras, es innombrable. Aunque es probable que las salchichas salgan mucho mejor libradas que las leyes, especialmente las colombianas.

En la pléyade de intereses individualistas del Congreso Colombiano, se planean y hacen las grandes normas que regulan nuestra existencia.
Todo está reglado. Desde antes de nacer incluso ya se estatuyó cuándo y a partir de qué instante se es "persona humana" en el Código Civil. Cómo nacer, cuánto pagar por ello, quién pagará por ello, para qué nacer, dónde estudiar, cómo estudiar, como evaluar ese estudio, cuántos hijos tener, dónde vivir, cuánto pagar por vivir, cómo vivir, para qué vivir y en caso del infortunio de caer en enfermedad, ya está la ley que regula la enfermedad y el tratamiento y que según la Constitución Nacional es la solución al problema de la enfermedad, la ya mencionada Ley 100 de 1993. Cómo enfermarse, dónde enfermarse y cómo sufrir la soledad y la indiferencia de tener que morir cuando la institución lo determine pertinente. Porque un enfermo básicamente le cuesta caro a la institución médica y al Estado.

\section{BIOPOLÍTICA}

La norma que siempre se presenta después del hecho social, se encuentra acompañada de tres tendencias que surgen después de la norma, una es la Bioética, que empieza a susurrar relevantes aspectos de la ética que deberían acompañar todas las discusiones de las actuaciones científicas y normativas que implicaran la administración de la vida. La Biojurídica que empieza a tomar el eje central de las discusiones de las normas que regulan todos los aspectos de la vida y la muerte de un individuo. Y una tercera forma de ver el espectro que es ahora de las más importantes es la Biopolítica., que ya contempla aspectos económicos y políticos dentro de la norma y en general de la sociedad.

Acercándose a una definición de Biopolítica, se podría afirmar que es la política de la vida. El biopoder es su brazo activo y en este sentido se puede afirmar que biopoder es un término originalmente acuñado por el filósofo francés Michel Foucault ${ }^{2}$ para referirse a la práctica de los Estados

2 "Michel Foucault, 1926-1984, fue un filósofo francés, que procuró mostrar que las ideas básicas que la gente considera verdades permanentes sobre la naturaleza humana y la sociedad cambian a lo largo de la historia. 
modernos de "explotar numerosas y diversas técnicas para subyugar los cuerpos y controlar la población" (Foucault, 1986). Foucault introdujo este concepto en la obra El poder del conocimiento (The will of knowledge) ${ }^{3}$, el primer volumen de su Historia de la sexualidad (History of Sexuality).

Castro (2010, p.9) afirma que en el curso Seguridad, territorio y población, Foucault evidencia su intensión de develar el significado del término "Biopolítica", pero que durante su desarrollo, termina ahondando en el análisis de lo que él llama "dispositivos de seguridad". Foucault establece que el surgimiento del biopoder empieza en el análisis de la ruptura del poder soberano francés del siglo XVIII y la puesta en escena de nuevas formas de poder en el XIX. El biopoder busca la administración de la vida, volviéndola un objeto susceptible de manipulación y conducción por parte de las diversas relaciones de fuerza que intervienen dentro de la sociedad, en últimas, el poder.

De este modo, se distinguen dos conjuntos de técnicas de biopoder que surgen en los siglos XVII y XVIII. La primera es la anatomopolítica ${ }^{4}$, caracterizada por ser una tecnología individualizante del poder, tiene como objetivo examinar los comportamientos del individuo y su cuerpo, con el fin de anatomizarlos, es decir, producir la obediencia y la fragmentación de los cuerpos. En esta tecnología el control del cuerpo se fundamenta en la disciplina y toma como herramientas de control a la vigilancia, el control, intensificación del rendimiento, multiplicación de capacidades, el emplazamiento, la utilidad, etcétera. (Foucault, 2007).

Sus estudios pusieron en tela de juicio la influencia del filósofo político alemán Karl Marx y del psicoanalista austriaco Sigmund Freud.

3 Michel Foucault escribió tres volúmenes de obras de 1976 a 1978 The Will to Knowledge (Histoire de la sexualité, 1: la volonté de savoir), originalmente en francés. The Use of Pleasure (Histoire de la sexualité, II: l'usage des plaisirs), and The Care of the Self (Histoire de la sexualité, III: le souci de soi).

4 Término también acuñado por Foucault que se usa para determinar el poder que asiste al soberano para reglar sobre todos los aspectos de la humanidad del individuo
El segundo grupo de técnicas de poder es la biopolítica y tiene como propósito la gestión de las poblaciones humanas, como grupos de seres vivos sometidos a leyes biológicas. Es en este sentido en donde cobra importancia la obra Nacimiento de la Medicina Social, en donde se establecen las primeras formas de evaluación de la fuerza activa de la población tales como las tasas de natalidad, mortalidad, morbilidad, movilidad en los territorios, por medio de las cuales se busca su control y conducción (Foucault, 1977 ).

De este modo, según la perspectiva foucaultiana, el poder se torna materialista y menos jurídico, ya que ahora debe tratar respectivamente, a través de las técnicas señaladas, con el cuerpo y la vida, el individuo y la especie.

La valoración que hace Foucault en torno al biopoder y sus técnicas es el hecho de que la vida ha sido gobernada, manipulada, controlada, vigilada y medida por el poder, y este fenómeno ha facilitado la acumulación de capital como la expresión más evidente de la ideología del capitalismo en la sociedad contemporánea insertando a los cuerpos dentro del aparato productivo, cosificándolos y determinándolos como objetos económicos.

En cuanto a la sociedad del miedo, se produce cuando, todas estas técnicas y estrategias políticas: la anatomopolítica en un principio y la biopolítica logran su objetivo de controlar y regir hasta el aspecto más íntimo de los individuos. Se comienza entonces, citando algunos planteamientos que permiten al lector acercarse al concepto de Sociedad del miedo a través del enfoque que le han dado las principales mentes que han procurado capturar el criterio que caracteriza las sociedades paralizadas, como la de Colombia, compuesta por individuos indiferentes al dolor ajeno aunque totalmente intolerantes a los derechos fundamentales del otro.

\section{LA NORMA Y EL MIEDO EN LA SOCIEDAD DEL CONTROL}

La norma ha generado a lo largo de los siglos, una serie de miedos que entre represados y latentes, 
se encuentran en el corazón del hombre. En una sociedad disciplinaria, tal y como lo establece Santurio (2007), el miedo se presentaba en los procesos de comunicación de los códigos de conducta, se establecía en las instituciones representativas de la soberanía del Estado y también en lugares confinados (hospitales, cárceles, etcétera).

Ya en la sociedad del control ${ }^{5}$ no tiene una aplicabilidad ni en tiempo ni en espacio físico, se caracteriza por recorrer todo el tejido social en el cual se origina y se convierte en un mecanismo disponible para todos los agentes generadores de cultura. El Estado ha sabido aprovechar todos los mecanismos disponibles para reglar absolutamente todos los aspectos de la vida del hombre, en procesos de gerenciamiento y administración de la vida a través de la norma.

\section{BIOPOLÍTICA Y BIOJURÍDICA, LA ECONOMÍA DE LA ENFERMEDAD A LOS OJOS DEL FISCO}

En el caso de la enfermedad y los tratamientos vitales, las erogaciones y costos de las medicinas y procedimientos en casos de enfermedades graves, son bastante elevados. El dinero que se gasta en el mantenimiento de un paciente terminal, se necesita para cosas más importantes. Hoy en día en el planeta hay demasiados seres humanos, la población mundial aumenta ${ }^{6}$, sobre todo en países en vías de desarrollo, a tasas alarmantes. La ley 100 de 1993 con sus figuras monstruosas de EPS y medicina pre pagada, hacen cada vez más pesada la carga de los que pagan y no usan los servicios y más gravosa la de los que necesitan

5 La sociedad del Control es un concepto desarrollado por teóricos posmodernos, en el cual, las comunicaciones y la tecnología se configuran como el modelo de creación de subjetividades (creación cultural), es una sociedad que se caracteriza por la carencia intencionada de medidas legales que controlen el abuso de las tecnologías.

6 El 30 de octubre de 2011, con el nacimiento de Danica Mae Camacho en Manila, Filipinas se alcanzó la cifra de 7 mil millones de habitantes. Fuente: Llega al mundo el habitante 7 mil millones» (en español). Consultado el 18 de marzo de 2012. tratamientos, medicinas y cuidados, y poco dinero aportan al sistema. Todos están inconformes, hasta los profesionales de la salud, los enfermos y los cotizantes. Esas son las consecuencias de imitar sistemas que ya han fracasado en otros países, y de traerlos a Colombia sin un estudio previo de conveniencia y de factibilidad.

Retomando el Derecho y la norma que regula nuestras vidas, concretamente el tema de la enfermedad y la muerte, llega el tema de la Bioética, la biopolítica y concretamente la biojurídica. Los cambios de paradigma, dentro de los cuales ahora se considera que todo debe estar reglado por el Derecho, incumben también a la Bioética, que a veces se pronuncia sobre aspectos que hacen parte del fuero interno y que deberían dejarse allí, sin regla jurídica. No todas las normas reflejan comportamientos deseables, o moralmente aceptables; pero también es cierto lo contrario no todo comportamiento moral, ético o deseable tiene su norma que garantice su obligatorio cumplimiento. (Broekman, 1998)

Ya se desbordó la órbita de acción de la relación médico-paciente. Hoy en día hablamos de estructuras más grandes, que involucran aspectos económicos como primordiales, sin serlo, y que abarcan el tratamiento médico dando lugar a juicios bioéticos. En últimas dejando de lado el tratamiento clínico efectivo, el desarrollo de la medicina en cuanto tal trae consigo consideraciones bioéticas que hay que discutir y que en la mayoría de los casos, carecen de la fuerza vinculante para ser acatadas, porque la norma está por encima de los comportamientos individuales.

Los temas que hay que discutir en torno a la medicina actual traspasan las fronteras de lo médico y muchas veces se posan en el ámbito de lo financiero, incluyendo la asignación de recursos para mantener un sistema, que en el caso del colombiano, como todo lo que se adopta en Colombia a través de normas, es imitación de sistemas foráneos implantados sin el lleno de requisitos culturales.

Las normas y los grandes sistemas legislativos (como el sistema penal acusatorio) que se han 
"importado" para engrosar el régimen legislativo colombiano, han carecido de un estudio previo de factibilidad y de conveniencia en donde la idiosincrasia sui generis del colombiano haya sido tenida en cuenta. Las pequeñas características que hacen parte de una cultura como la colombiana, marcan las grandes diferencias por las cuales fracasan los sistemas legales importados a pupitrazo en este país. Como la ley general de educación y en concreto la ley general de salud $y$ enfermedad que hemos mencionado.

Los desarrollos del Derecho en torno a estos aspectos éticos y médicos, no obedecen solamente a un cambio de marco institucional o a una expansión de la medicina y la sociedad. Sino que obedecen principalmente a un nuevo enfoque, un nuevo paradigma y una nueva determinada imagen del hombre ${ }^{7}$. Tal es así que el ejemplo claro es que ahora, no solamente en la norma sino en la academia y en cualquier conversación desprovista de formalismos, cuando se menciona al hombre hay que decir "y la mujer" también". Acaso por hilar más delgado y procurar aparentar ser más incluyente, al redactar una norma el Estado se acerca más al deber ser y a la perfección? Esto es una falacia. Una cosa es la semántica y otra cosa la realidad.

Lo único cierto es que al igual que la imagen de belleza de mujer que tuvo Renoir, Velásquez, Da Vinci y otros maestros de diversas épocas de la humanidad; hoy en día esa imagen ha cambiado, y dista por varios kilos, entre otras cosas, de la imagen de belleza insulsa y lapidaria que nos ha querido vender Hollywood de la mujer anoréxica, pálida y ojerosa. El ideal de mujer hoy día, que se adentra en los corazones de los hombres y de tanta adolescente que en el intento de alcanzarla,

7 Entiéndase por tal el concepto de persona, incluso el que trae el Código Civil Colombiano en su artículo 73. Que es copia del Código Civil de Chile, que no fue más que la traducción del Código de Napoleón Bonaparte de 1804.

8 Véase por ejemplo la norma de que regula aspectos de la infancia en Colombia, y ese era su nombre. Hoy en día debió cambiar su nombre y procurar ser inclusiva al enunciar "niños, niñas y adolescentes". no logra darse cuenta a tiempo, que solamente después de varias semanas después de muerta, alguien logra lucir como la imagen anhelada de belleza, la extrema delgadez, las orejas y el pálido destello de la pancarta del producto de belleza de moda. La cultura se transforma y con ella se transforma nuestro morir. (Broekman, 1998)

También la imagen de persona ha cambiado desde los griegos, como ha cambiado la ley que determina los alcances de la persona, en lo atinente a su vida, su muerte y la enfermedad.

Primero en la vida se da el hecho, luego la norma y luego el pronunciamiento bioético. En consecuencia podemos afirmar con Broekman (1998, pg. 11) que todos los desarrollos médicos van acompañados de desarrollos jurídicos, que no necesariamente corresponden a desarrollos de la bioética. Cada vez más formas de pensamiento juridiformes, prevalecen sobre los desarrollos médicos y éticos. Tal es el caso de la eutanasia del cual nos ocuparemos a continuación.

\section{EUTANASIA, UN LUGAR PARA LA MUERTE EN LA VIDA}

\section{"La vida sin muerte es como realidad sin sombra" George Bataille}

Al parecer siempre nos ocupamos de la muerte de los demás, nunca de la nuestra, porque es mucho más difícil imaginar nuestra propia muerte sobre todo en circunstancias en las que un tercero debe acabar con ella; nuestra capacidad de compasión no da para tanto. (Broekman, 1998, p. 181).

La bioética y particularmente la ética médica, tratan la eutanasia como un cuadro clínico. La eutanasia, implica la actuación siempre de un tercero y ese tercero debe ser calificado del sector de la salud. Jamás un médico o un enfermero imaginan su propia muerte, esa situación le resulta inimaginable, les haría un poco más débiles entonces. La problemática de la eutanasia demuestra que es un reflejo fiel del 
espejo de la sociedad vigente en ese momento en una cultura determinada.

La eutanasia como fenómeno, se ha practicado más ampliamente en la época contemporánea, a pesar de tener dos raíces griegas bien definidas "eu" y "thanatos", buena y muerte. Es una creación artificial reciente, ya se explicó, que no existía en Griego antiguo, ni en la conciencia desarrollada en ese momento. Aunque sí había sentimientos en la Grecia Antigua, relacionados con "el buen morir" que supone un fallecimiento acaecido en buenas circunstancias sociales y morales. La finalidad del ser humano era, de acuerdo con ese sentimiento de vida, cuidarse bien y correctamente. Esa idea fue fundamental para Hipócrates, quien compartía este concepto con los antiguos Griegos que hablaban más de cuidarse que de curarse (Broekman 1998, p. 183).

Hoy en día la palabra eutanasia, pone en evidencia un pensamiento enteramente distinto en el que "la muerte buena" es simplemente el resultado inmediato del actuar de otra persona, lo que la diferencia del suicidio. Y que se puso de moda, fue en la segunda guerra mundial, en donde los más destacados doctores, aplicaban esa "buena muerte" a cientos y miles de personas, que simplemente no era conveniente mantener en el régimen. Hoy en día es también una acepción con matices económicos la eutanasia. Cuánto le cuesta al Estado mantener a un enfermo terminal? La medicina está para sanar? Para mantener por la mayor cantidad de tiempo vivo a alguien? O acaso la medicina es una de las fichas más relevantes de un sistema jurídico legal que procura que las arcas del Estado, a pesar de la corrupción y otros escapes contra los que no vale la pena luchar, permanezcan llenas de dinero para que el gobernante de turno tenga con qué llenar el tanque de gasolina de su carro?

\section{HOMICIDIO, ACTUACIÓN MÉDICA Y AUTONOMÍA}

Cuando el tercero que aplica la eutanasia no es un médico, estamos frente a un homicidio y es penalizado por el Estado. Eso dice la mayoría de Códigos Penales del mundo. Es el suicidio acaso una forma de eutanasia en la cual autorizamos al médico para terminar con nuestra vida?

La eutanasia hoy como los principales temas de la bioética, es más un debate político, o quizás hasta biopolítica, entendiendo por tal aquel que sostienen quienes detentan el poder y tienen la autoridad para crear las normas de gerencia de la vida, que un debate humano, filosófico y ontológico del hombre común que quisiera vivir mejor en todos los aspectos incluso en el de la muerte, ya que ese hombre se encuentra ocupado procurando sobrevivir en un Estado y en un planeta lleno de normas que restringen en todos los ámbitos su actuar. Puede el hombre por sí mismo determinar el papel que le corresponde en la naturaleza? Por supuesto que no, a no ser que tenga la autoridad de pertenecer a la élite fortuita que hace las normas en un Estado. Pero generalmente un hombre que tenga estos ideales trascendentes no aspirará a cargos políticos que son generalmente obtenidos contra todo principio o norma ética y moral trascendente. Los engranajes políticos existen y es de necios negarlos. (Guerra, Y. 2009)

Hay diferencia entre tratamiento paliativo y tratamiento eutanásico?, ambos tienen finalidades diferentes. La confrontación entre "querer cuidar" $y$ "querer dejar en libertad" a menudo encuentra su base en la autodeterminación. La interpretación y el empleo práctico de ese concepto dejan ver las insalvables diferencias entre cuidar y dejar en libertad a alguien ya que mediante el ejercicio íntegro de sus derechos el interesado desea aportar una interpretación solitaria y fuertemente individualista a la autodeterminación (Broekman, 1998, p. 187). Por ello el lema "la muerte como una decisión es tan problemático". Porque no hay derecho sin el reconocimiento de la legitimidad de ese derecho que lo da precisamente estar inmerso en una sociedad que acata las disposiciones de las normas que son en principio, para todos.

O bien se hace primar la vida sobre la autodeterminación de un individuo o bien se hace primer ésta sobre la vida.. Cuál es más relevante? 
A veces la respuesta tiene que ver con lo que más le conviene al Estado, y todo podría reducirse a un asunto de economía pública. Cuánto le cuesta al Estado mantener (brindar tratamiento médico) durante años a una persona en estado vegetativo? Cuál es la conveniencia de esto?

La presencia de la tensión entre el reconocimiento del "yo humano" que prima sobre un concepto biomédico de la "vida", y el reconocimiento de la supremacía de este concepto biomédico de la "vida" sobre el "yo humano". (Broekman, 1998).

\section{DERECHO Y MEDICINA}

De las consideraciones anteriores se desprende que eutanasia se desarrolla dentro de un complejo entramado de consideraciones jurídicas y médicas sin tener en cuenta el marco moral, psicológico ideológico de esa red.

De allí la idea con la que se empieza este ensayo, desde antes de nacer todas las actuaciones de una persona están regladas por el Derecho, y esta la de la eutanasia es también una discusión jurídica y política, en la que tiene su asidero también el aspecto económico de las arcas del Estado y de cuánto le cuesta al Estado brindar tratamiento paliativo o eutanásico a un individuo promedio. ${ }^{9}$

Si se tratara exclusivamente de un asunto jurídico, Broekman remite al médico al asunto de la buena fe como el principio fundamental que regula las actuaciones en el Derecho (Broekman, 1998). Esto es cierto si se tratara "solamente" del hecho de determinar si se hace el mejor esfuerzo para mantener con vida a una persona, y se excluye el criterio económico, de cuánto cuesta brindar tratamiento paliativo a un enfermo terminal o a un paciente en estado vegetativo. Suponemos que solamente la jurisprudencia en cada caso dilucidará, ya a futuro, porque al caso que le dio

9 Individuo promedio es cuando no se trata de una figura pública, como en el caso de Gustavo Ceratt, famoso cantante Argentino que tuvo sus inicios en la banda de Rock Latino Soda Estéreo; y quien hoy en día se encuentra en estado vegetativo desde el año 2010. origen al proceso que redundó en la sentencia sería inocuo, quien determinará qué tener en cuenta según el momento que esté viviendo un Estado, según las condiciones elementales de tiempo, modo y lugar del mismo.

La norma no debería cambiarse para reflejar circunstancias particulares, se entiende que lo que brinda estabilidad y certeza, más allá de seguridad, en la mente de los administrados, es la permanencia de la norma en el tiempo, independientemente de las circunstancias de cada caso en particular. Pero es la jurisprudencia la que entraría a determinar si la eutanasia de los enfermos terminales o en estado vegetativo, de un Estado en guerra y pobre, podría considerarse conveniente o no.

La eutanasia, no supone un tratamiento médico complejo. En términos médicos la intervención eutanásica apenas tiene que ver con la compleja técnica médica moderna. Esa sencillez técnica contrasta con la complejidad de la calificación médica y jurídica del tratamiento eutanásico. En términos médicos la diferencia entre una o dos inyecciones de morfina es una pequeñez, para lo que sería la consecuencia, entre la vida o la muerte de un individuo (Broekman, 1998).

\section{CONCLUSIONES}

Todas las actividades del hombre están regladas por la norma jurídica y por el derecho a través no solamente de la ley sino además de relaciones de poder donde el Estado prevalece e impone su voluntad por encima de la conveniencia del ser humano, teniendo además en cuenta un factor preponderante y de vital importancia: la economía y el presupuesto del Estado, sobre todo en cuestiones de salud, enfermedad, muerte y vida.

La vida de un ser humano, tiene más de trascendente que de biológico y entender la vida de un individuo a través de procesos mentales solos, en el mero criterio del zoe de Aristóteles es un error.

Cuestiones de ética médica deben ser entendidas en la tradición del Galeno de Pérgamo, el eminente médico romano de origen griego, quien destacó 
que el cuerpo humano emite señales y produce símbolos específicos con respecto a su salud y su bienestar. Los médicos tienen que leerlos, interpretarlos y crear su diagnóstico y terapia acorde con ellos. Leer e interpretar, por tanto, eran en esa época, una obligación primordial dentro de las funciones de cada médico. Muchos de los significados encontrados por la medicina, así entendida, fueron los que construyeron el gran cuadro de nuestro paisaje espiritual y material como humanos. Dentro de los dibujos de líneas y signos, el acto final de gerencia de la propia existencia es el de la autorización de eutanasia, muerte a manos de un tercero calificado. Que tiene como relevante el elemento de tercero calificado (en caso contrario y si el tercero no es calificado, se trataría de un homicidio) y que se trate de un tercero, de no ser así estaríamos hablando de un suicidio.

Se pueden plantear numerosas discusiones sobre este tema, pudiendo distinguir diversos aspectos del entramado: clases de eutanasia, pasiva y activa; la autonomía del paciente la economía del Estado la naturaleza de la vida, pero por sobre todas las cosas, siempre prevalecerá el aspecto de la norma y del Derecho para determinar los aspectos de la vida, la muerte y la enfermedad de todo individuo.

Vale la pena concluir preguntando, si existe un lugar en nuestra sociedad para un saber ético que sea escuchado y compartido? No debería acaso en el Congreso de la República existir un lugar en que las actuaciones sinceras de los hombres den cuenta de sus intenciones y actitud moral? A qué clase de discurso pertenece la ética si no tiene fuerza vinculante? Sobre cuáles estructuras de la realidad se erige el discurso de la eutanasia $y$ de la salud y la vida en general? O es acaso el discurso de la ética un discurso impotente sobre todo frente a la norma ya creada?

En lo atinente a la eutanasia cada caso es especial y debería discutirse teniendo en cuenta el elemento conveniencia para el paciente, dolor, sufrimiento y perspectivas de futuro inmediato. El dinero y los intereses de los demás deberían ser aspectos no relevantes, especialmente si ese tercero es el Estado y se manifiesta a través de las normas.

\section{REFERENCIAS}

Agamben, G. (2009). La Nuda vida y el Homo Sacer.

Bauman, Z. (2007). Miedo Liquido: La sociedad Contemporánea y sus temores. Barcelona. Ediciones Paidos Ibérica S.A

Barthes, Roland. (1953). El grado cero de la escritura.

Beck, U. (1992). Risk Society: Towards a New Modernity. London. Editorial Sage 1992.

Beck, U. (2002). La sociedad del riesgo global. Madrid. Siglo XXI Editores.

Beck, U. (2008). La sociedad del riesgo mundial. En busca de la seguridad perdida. Barcelona: Ediciones Paidos Ibérica S.A.

Broekman, Jan. (1998). Bioética con rasgos jurídicos. .Editorial Dilex. Madrid

Cántaro, A .(2006). Europa soberana: la Constitución de la Unión entre guerra y derechos. Mataró. Ediciones El Viejo Topo.

Castro, S. (2010). Historia de la gubernamentalidad: razón de estado, liberalismo y neoliberalismo en Michel Foucault. Bogotá. Siglo del Hombre Editores.

Díaz, Pedro Agustín. (2009). Estado y tercer mundo, el Constitucionalismo. Editorial Ibañez. Bogotá.

Fazio, M. (2004). Gobernar los riesgos. Ciencia y valores en la sociedad del riesgo. Organización de Estados Iberoamericanos para la Educación, la Ciencia y la Cultura (OEI). 
Foucault, Michel. (1986). El Cuidado de sí. (Título alternativo, Historia de la sexualidad volumen III. Madrid, Editorial Siglo XXI.

Foucault, M. (1976). Vigilar y Castigar. Bs. As. Siglo XXI Editores.

Foucault, M. (2003). Hay que defender la sociedad. Madrid. Ediciones AKAL.

Foucault, M. (2007). El nacimiento de la biopolítica. Buenos Aires Fondo de Cultura Económica

Foucault, M. (1977). Nacimiento de la Medicina Social. Conferencia El nacimiento de la medicina social - Revista centroamericana de Ciencias de la Salud.

Guerra, Y. (2009). Liderazgo, una propuesta pedagógica eficaz. UMNG.

Hamer, Dean. (2004). EL Gen de Dios. The Biology of Belief.

Korstanje, M. (2010). El miedo en el nuevo milenio: un abordaje antropológico para comprender la postmodernidad, Edición electrónica gratuita.

Korstanje, M. (2010). El miedo político en C. Robin y M. Foucault. International Society of Philosophers, United Kingdom. Paidos.

Lara, J. D. (2009). La sociedad del miedo edificada por el desarrollo modernista: enlaces con la fragilidad humana y la percepción del otro. Universidad de Puebla. En: Nómadas. Revista Crítica de Ciencias Sociales y Jurídicas.
O' Donnell, P. (2009). La sociedad de los miedos. Buenos Aires. Editorial Suramericana.

Robin, C. (2004). Fear The history of a Political Idea. Oxford University Press. Ei

Román, J. (2006). La sociedad del miedo.

Salazar, R. (2010). Los Miedos ocultos en la sociedad del Siglo XXI. México. Universidad Autónoma de Sinaloa.

Santa Cruz, E. (2010). La administración mediática del miedo.

Santurio, R. (2007). La sociedad del Miedo de los Medios.

Tirado, M, "Algunas reflexiones sobre la aplicación de los principios de la bioética en el ordenamiento jurídico colombiano a la luz de los derechos fundamentales: alcance jurisprudencial sobre la vida y el derecho a la vida" En: Colombia Revista Latinoamericana De Bioetica/Latinamerican Journal Of Bioethics ISSN: 1657-4702 ed: v.11 fasc.1 p.110 - 120 ,2011 Ordoñez, L (2006).

La globalización del miedo. En: Revista de Estudios Sociales No 25. Bogotá. Universidad de los Andes. Facultad de Ciencias Sociales.

Willer, R. (2004, Septiembre) "The Effects of Government-Issued Terror Warnings on Presidential Approval Ratings" Current Research in Social Psychology, 10, 1. 